\title{
Collagen structure changes during chrome tanning in propylene carbonate
}

\author{
Yi Zhang ${ }^{1}$, Jenna Kate Buchanan ${ }^{1}$, Geoff Holmes ${ }^{1}$, Bradley William Mansel ${ }^{2}$ and Sujay Prabakar ${ }^{1 *}$ (D)
}

\begin{abstract}
Green solvents, such as propylene carbonate (PC), can be used in leather processing to improve the efficiency of chrome tanning and reduce wastewater. Here we report a combined small-angle $X$-ray scattering (SAXS) and differential scanning calorimetry (DSC) study on PC and its efficacy as a carrier medium during chrome tanning. SAXS analysis on the collagen structure of chrome tanned leather using PC, compared to conventionally tanned leather using water, showed an increase in $\mathrm{Cr}$ uptake in addition to the more uniform penetration of $\mathrm{Cr}$ through the leather cross-section. The increased binding of $\mathrm{Cr}$ to the collagen matrix drives the decreased hydration environment of the collagen triple helix. Furthermore, DSC studies show a uniform hydrothermal stability for the PC samples due to the more even distribution of $\mathrm{Cr}$ through the collagen matrix. Understanding the mechanisms by which chrome tanning occurs in non-aqueous solvents can guide us towards a more sustainable future for the leather industry.
\end{abstract}

Keywords: Propylene carbonate, Collagen structure, SAXS, DSC, Chrome tanning

\section{Introduction}

The increasing demand for the leather industry to adopt cleaner carrier mediums due to strict environmental legislation has led researchers to search for non-aqueous alternatives such as those listed in the GlaxoSmithKline (GSK) solvent selection guide [1-3]. While the role of water in tanning is crucial and should not be underestimated $[4,5]$, wastewater management [6] and efficient $\mathrm{Cr}$ uptake are issues that still need to be addressed [2, 3]. Non-aqueous solvents are promising alternatives to water treatments as they may allow for $100 \% \mathrm{Cr}$ uptake and can be more easily recycled, significantly reducing the wastewater produced in the tanning process $[2,3,7,8]$. Recently, Rao et al. identified the use of many non-aqueous carrier mediums, in particular propylene carbonate, because of its ability to carry out zero discharge tanning with high $\mathrm{Cr}$ uptake and comparable hydrothermal stability with conventional leather [7, 9]. Propylene carbonate is a dipolar aprotic solvent which is nontoxic, biodegradable and classified as a volatile organic compounds (VOC) exempt green solvent by the environmental protection

\footnotetext{
* Correspondence: sujay.prabakar@lasra.co.nz

${ }^{1}$ Leather and Shoe Research Association of New Zealand, P.O. Box 8094,

Palmerston North 4472, New Zealand

Full list of author information is available at the end of the article
}

agencies of the United States and Europe [7, 10]. When used as a medium for the chrome tanning process, $\mathrm{PC}$ has been shown to be recyclable for use in successive tanning batches without any pre-treatment [7]. However the exact mechanism by which chromium sulphate interacts with the collagen molecule in the absence of an aqueous solvent is yet to be investigated.

The mechanism of chrome tanning relies on the formation of a trimeric chromium aquo complex in unbound water before cross-linking with the carboxyl group of the collagen [11]. Chromium is known to interact with the telopeptidyl aspartic and glutamic acid residues in collagen affecting its intermolecular structure. Collagen molecules are aligned in a quarter stagger structure, resulting in repeating gap/overlap regions within the fibrils $[12,13]$. Because of its ordered arrangement, molecular level information can be acquired by $\mathrm{X}$ ray scattering techniques, including diffraction, to measure the periodicity of the gap/overlap regions ( $D$-period) and the size of collagen fibrils [14-19]. The interactions between water and collagen play a crucial role in the stability of the triple helix [20-22]. Changes in the solvent used in leather processing can affect the hydration of collagen and in turn affect its intermolecular structure. In this study we use SAXS and DSC to investigate the 
molecular level changes in collagen structure during chrome tanning with a non-aqueous green solvent such as PC. SAXS measurements provided information on the changes in axial periodicity and dehydration through the leather cross-section and were analysed in conjunction with $\mathrm{Cr}$ uptake and the onset temperature of collagen denaturation.

\section{Experimental}

\subsection{Materials}

Pickled grain splits of cattle hide were received from local tannery and transported to the New Zealand Leather \& Shoe Research Association (LASRA), Palmerston North for processing in the pilot-tannery. Propylene carbonate (99.7\%) from Carl Roth $\mathrm{GmbH} \& \mathrm{Co} . \mathrm{Kg}$, Germany, Chromosal ${ }^{\circ} \mathrm{B}\left(25 \% \mathrm{Cr}_{2} \mathrm{O}_{3}, 33 \%\right.$ basicity) from Lanxess, Germany and sodium bicarbonate from SigmaAldrich was used without further modification.

\subsection{Leather sample preparation}

Pickled $(\mathrm{pH}=3)$ grain split bovine hide was treated with basic chromium sulphate $\left(25 \% \mathrm{Cr}_{2} \mathrm{O}_{3}, 33 \%\right.$ basicity) at room temperature for $6 \mathrm{~h}$ at different concentrations ( $2 \%, 4 \%, 6 \%$ and $8 \%$, wt $\%$ of the hide) in water or PC with same weight as the hide. After the treatment, leather samples were stored at $4{ }^{\circ} \mathrm{C}$ prior to SAXS analysis, and the tanning solutions were collected for analysis of $\mathrm{Cr}$ uptake using atomic absorption spectrometry (AAS).

\subsection{Small-angle X-ray scattering (SAXS) measurements}

Untanned hide and chrome tanned leather samples were cut into strips of size $20 \mathrm{~mm} \times 1 \mathrm{~mm} \times 3 \mathrm{~mm}(\mathrm{~L} \times \mathrm{W} \times \mathrm{H}$, Scheme 1) and sealed using Kapton tape on both sides to keep the moisture levels constant. SAXS experiments were conducted on beamline 23A1 at the National
Synchrotron Radiation Research Centre (NSRRC) in Hsinchu, Taiwan. The measurements were carried out at a $3.232 \mathrm{~m}$ sample-to-detector distance with an X-ray energy of $15 \mathrm{keV}$. The scattering intensity $I(q)$ is presented as a function of scattering vector, $q$, with $q=4 \pi \sin (\theta / 2)$, where $\theta$ is the angle between incident and scattered radiation. The detailed diffraction peak fitting method is described in the Additional file 1. Relative diffraction peak intensity is calculated as $R_{i / j}=A_{i} / A_{j}$, where $A_{i}$ and $A_{j}$ stands for the area of peak order $i$ and $j$.

\subsection{Differential scanning calorimetry (DSC) measurements} DSC measurements were carried out on DSC Q2000, TA Instruments. Lyophilized samples were rehydrated as well as basified with $0.2 \mathrm{M}$ sodium bicarbonate solution to $\mathrm{pH}=4$, and then sealed in Tzero aluminium pans overnight, followed by running on the instrument at $5{ }^{\circ} \mathrm{C} /$ min from $30^{\circ} \mathrm{C}$ to $120^{\circ} \mathrm{C}$ under a $\mathrm{N}_{2}$ purge. Onset temperatures of the endothermic peaks on heat flow curves were taken as the denaturation temperature $\left(T_{d}\right)$ of the sample.

\subsection{Percentage uptake of chromium}

$\mathrm{Cr}$ uptake in each sample was measured by Varian SpectrAA 220FS atomic absorption spectrophotometer. The amount of $\mathrm{Cr}$ in the tanning solutions after the treatments were also measured using AAS. Hide samples and tanning solutions were treated with an excess of concentrated nitric acid, followed by a mixture of perchloric acid and sulfuric acid to solubilise $\mathrm{Cr}$ species. The solutions were then diluted with water and boiled again for $10 \mathrm{~min}$ to eliminate the residual oxidising acids. After cooling, the solutions were further diluted and filtered before measurement. The measurements were conducted

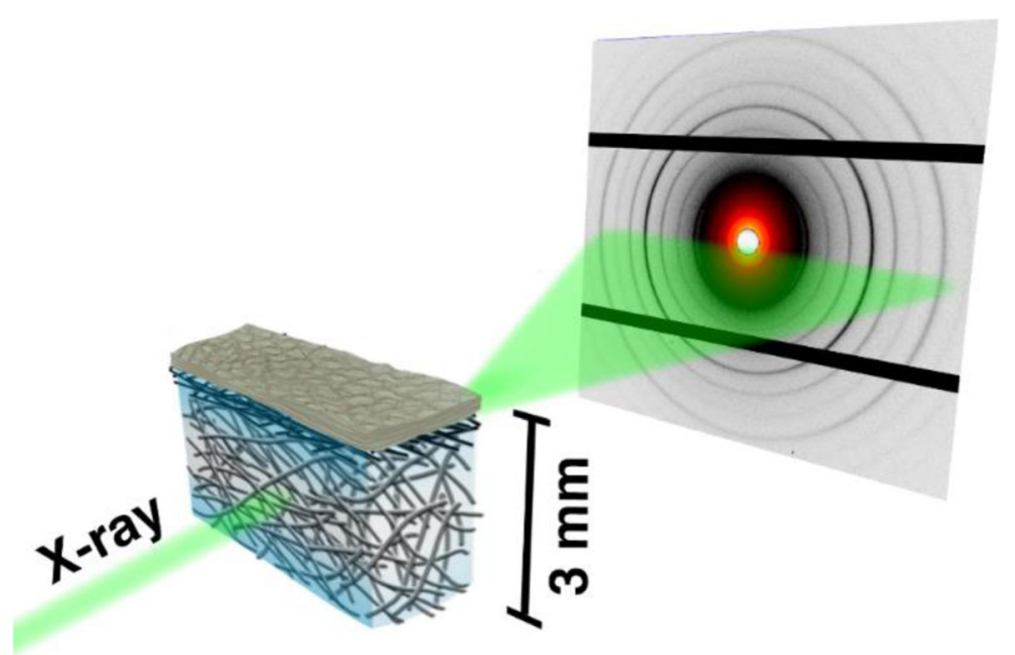

Scheme 1 Experimental setup for the SAXS measurements through the cross-section of bovine hide 
using an air/acetylene flame with a wavelength of 357.9 $\mathrm{nm}$ and a slit width of $0.2 \mathrm{~nm}$.

\section{Results and discussion}

\subsection{Chromium uptake from AAS studies}

To study the effect of propylene carbonate on the collagen structure of leather, we first treated pickled hide to both water (WT) and propylene carbonate (PC) based chrome tanning at increasing concentrations and studied their uptake using AAS (Table 1). The PC chrome tanned samples had very high uptake (almost 100\% within $6 \mathrm{~h}$ ), compared to the conventional chrome tanned samples $(<70 \%)$ and previously reported high exhaustion chrome tanning methods (up to $98 \%$ after overnight processing) [23-25]. Rao et al. observed similar results and attributed the drastic improvement in $\mathrm{Cr}$ uptake to the insolubility of chromium sulphate in PC which drives the $\mathrm{Cr}$ species into the collagen matrix by the mass transfer from the PC layer surrounding the surface of the hide to the minimal water in it $[7,9]$. While in water based systems, the uptake is driven by diffusion and is much slower $[7,9]$. Such a mechanistic difference results in the extremely high efficiency in uptake during chrome tanning in PC compared to aqueous systems.

\subsection{SAXS peak changes with tanning}

SAXS experiments were performed on both PC and WT samples (Fig. 1). To study the role of the solvent in $\mathrm{Cr}$ uptake as an event independent of fixation, we conducted SAXS measurements prior to basification. The characteristic scattering from the leather samples is from the long-range order of collagen molecules present in a quarter-staggered arrangement, with repeating gap/overlap regions in a characteristic axial periodicity ( $D$-period) $[16,17]$. The scattering profiles for pickled hide treated in both propylene carbonate (PC-0\%) and conventional (WT-0\%) solvents were similar to each other and exhibited the typical isotropic collagen X-ray diffraction patterns of bovine hide, displaying a series of Bragg peaks arising from the long-range ordered collagen structure [16]. On tanning with chromium sulphate (2\% to $8 \%$ ) for both PC and WT samples, significant changes in the relative diffraction intensities of the Bragg peaks were observed, indicating that the internal molecular structure

Table 1 Chromium uptake in propylene carbonate and water tanned samples obtained from AAS measurements

\begin{tabular}{lll}
\hline & \multicolumn{2}{l}{ Percentage uptake (\%) } \\
\cline { 2 - 3 } Chrome offer (\%) & Propylene Carbonate & Water \\
\hline 2 & $99.97 \pm 1.20$ & $69.09 \pm 0.21$ \\
4 & $99.99 \pm 0.70$ & $61.27 \pm 0.12$ \\
6 & $99.99 \pm 1.10$ & $56.28 \pm 0.39$ \\
8 & $99.99 \pm 0.80$ & $54.00 \pm 0.16$ \\
\hline
\end{tabular}

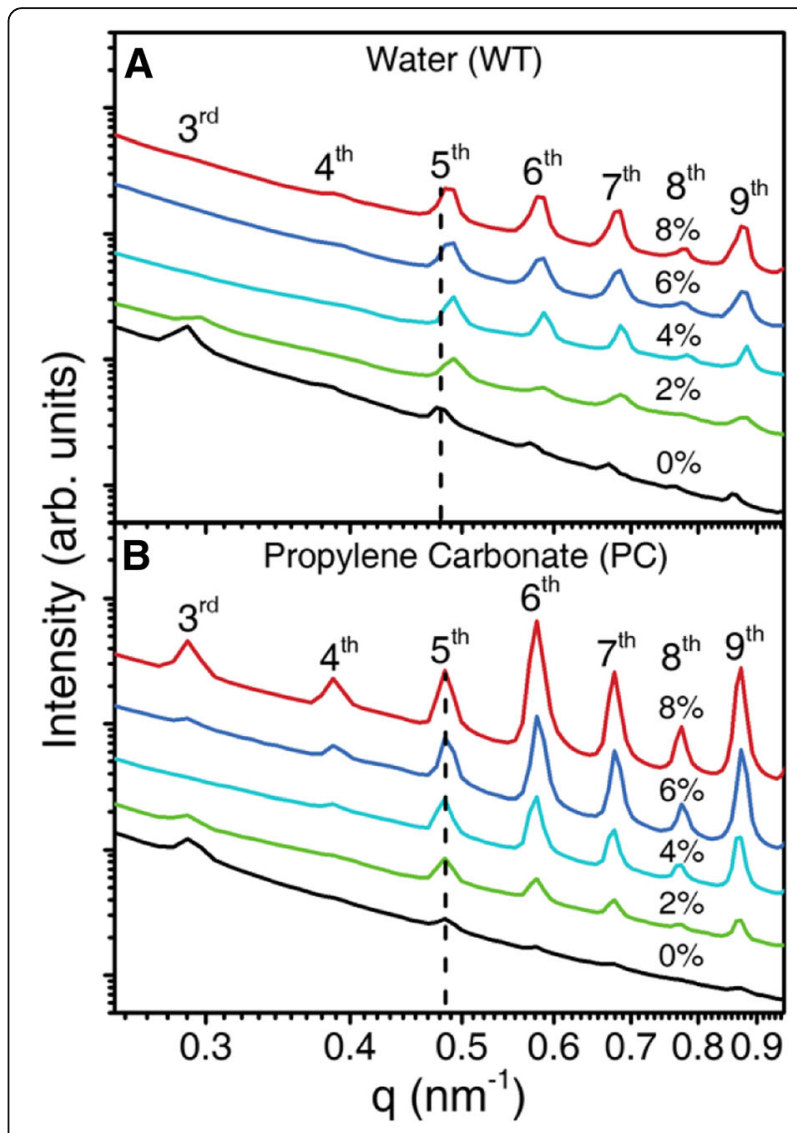

Fig. 1 SAXS data showing differences in scattering intensity between increasing concentrations of chromium sulphate for WT (a) and PC (b) based chrome tanning. Peaks correspond to $q=2 \pi n / D$ where $n$ is the peak order and $D$ is the $D$-period

of collagen had changed during tanning. This can be attributed to the uptake of $\mathrm{Cr}$ by the collagen matrix thereby enhancing the electron density contrast of collagen. The introduction of metallic species into the collagen matrix has previously been shown to cause an overall increase in the intensity of the Bragg peaks [26-28]. At concentrations of $4 \%$ to $8 \%$ chromium sulphate, the intensity of PC tanned samples (Fig. 1b) were observed to be higher compared to the WT samples, indicating a higher degree of uptake and binding with the collagen matrix. In the WT samples, a decrease in the average $D$-period with an increase in chrome concentration from $0 \%$ to $2 \%$ was indicated by the shift in the position of all diffraction peaks to higher $q$ for the WT-2\% sample (dashed line, Fig. 1). Interestingly, this is contrary to previous reports on chrome tanned hides and skins, where an increase in $D$-periodicity is observed [14, 18, 19]. This anomaly is ambiguous but we speculate that the discrepancy of $D$-period changes after chrome tanning emanates from the differences in the intrinsic collagen structure of the hides and skins $[13,19]$. The detailed mechanism of collagen $D$-period changes remains unclear, however, changes in $D$-period during 
chrome tanning can be attributed to the reaction of $\mathrm{Cr}$ species with telopeptidyl aspartic and glutamic acid residues that disturb the gap/overlap region of collagen [18]. By increasing the chrome concentration from $2 \%$ to $8 \%$, no further shift in peak position was observed, indicating the $D$-period was unchanged and that further binding of $\mathrm{Cr}$ in the telopeptidyl regions was no longer possible. In the PC samples, only a slight decrease in the average $D$ period was observed with an increase in chrome concentration from $0 \%$ to $2 \%$. This indicates that the binding of $\mathrm{Cr}$ to collagen in PC may be mechanistically different to that of conventional chrome tanning in water, resulting in only minor changes in the gap/overlap region of the collagen molecule.

\subsection{D-period changes through the leather cross-section}

To further understand the process by which $\mathrm{Cr}$ interacts with the collagen matrix in a PC medium, we studied the changes in $D$-period through the leather cross-section. In the WT samples (Fig. 2a), a decrease in the average $D$-period (through the cross-section) from 65.5 to $63.5 \mathrm{~nm}$ was observed with an increase in chrome concentration from $0 \%$ to $2 \%$. This observation suggests that when the chrome concentration is increased to $2 \%, \mathrm{Cr}$

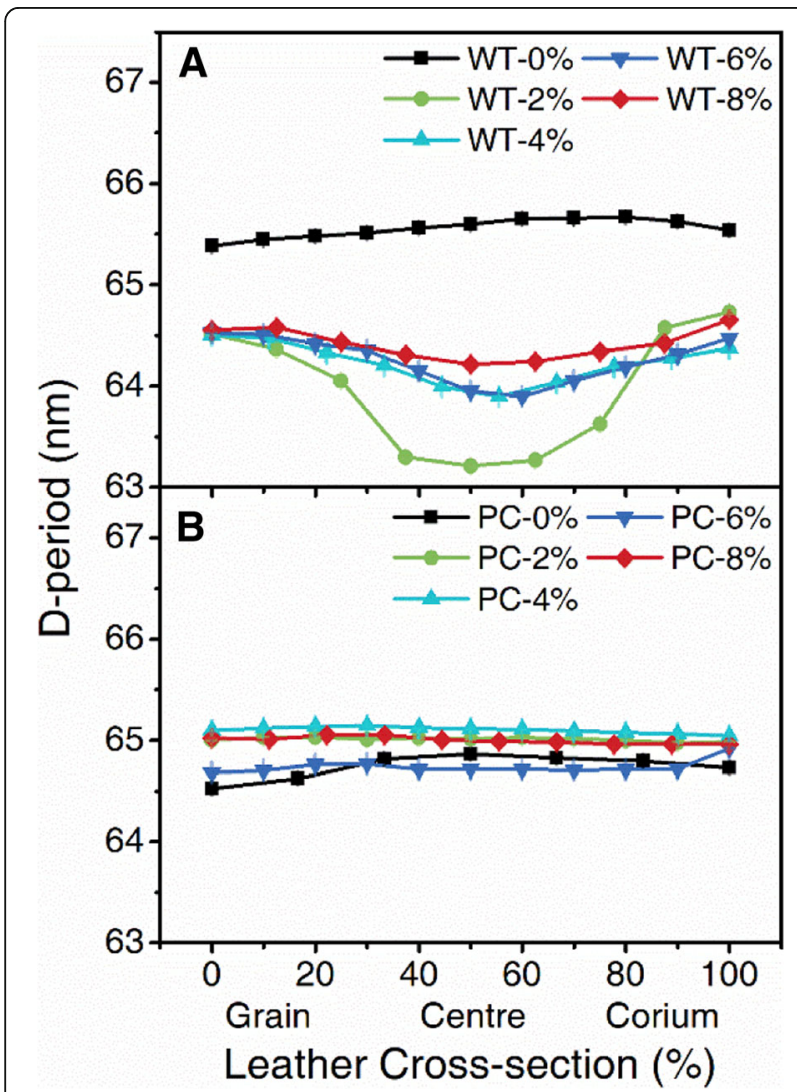

Fig. 2 D-period across the leather cross-section for increasing concentrations of chrome in WT (a) and PC (b) based chrome tanning reacts with the telopeptidyl aspartic and glutamic acid residues of collagen, changing the $D$-period. However, the $D$-period changes across the cross-section were observed to be non-uniform for the low concentrations to improve gradually at higher concentrations. We speculate that at low concentrations, surface (grain and flesh) fixation of $\mathrm{Cr}$ with the collagen molecules prevents the penetration of $\mathrm{Cr}$ into the centre region.

For the PC samples (Fig. 2b), the average $D$-period of untanned skin (PC-0\%) was observed to be much lower than the untanned WT samples (WT-0\%) and relatively uniform through the leather cross-section. On tanning with $2 \%$ chromium sulphate (PC-2\%), unlike the WT-2\%, no significant change in $D$-period through the cross-section was observed. However, the D-period was observed to be uniformly distributed through the leather cross-section. Further additions of chrome resulted in no change in $D$-periodicity but contrary to the WT samples, the axial periodicity for the PC samples were observed to be distributed evenly. This highlights the even penetration of $\mathrm{Cr}$ through the leather matrix when using a PC based chrome tanning process.

\subsection{Changes in $\mathbf{R}_{6 / 5}$}

The relative intensities of the 6 th/5th order peaks $\left(R_{6 / 5}\right)$ give an indication of the level of dehydration of the leather. Previous studies showed that the dehydration of fibrillar collagen resulted in an increase in intensity of the even-order peaks (particularly the sixth) and a decrease in the intensity of odd-order peaks [28-31]. In the WT samples (Fig. 3a), $R_{6 / 5}$ values showed non-uniformity across the leather cross-section, especially for $2 \%$ chrome concentration which exhibited a U-shaped curve. This non-uniformity improved with increasing concentration. There was a significant increase in the relative peak intensity for $2 \%$ to $4 \%$ chrome concentration, indicating the increasing dehydration of collagen due to $\mathrm{Cr}$ deposition. Chrome concentrations of $4 \%$ to $8 \%$ had similar $R_{6 / 5}$ values, suggesting there were no further changes in dehydration after $4 \%$.

For the PC samples (Fig. 3b), $R_{6 / 5}$ was observed to increase with chrome concentration. This indicated the dehydration of the collagen due to the deposition of $\mathrm{Cr}$ and displacement of water. $R_{6 / 5}$ values for PC were higher than WT for the corresponding concentrations, suggesting there was more water present in the WT treated leather compared to the PC samples. The greater dehydration of $\mathrm{PC}$ can be attributed to the higher $\mathrm{Cr}$ uptake for PC relative to the WT samples.

\subsection{DSC studies}

By studying changes in the hydrothermal stability, the effects of $\mathrm{Cr}$ binding to the collagen matrix can be 


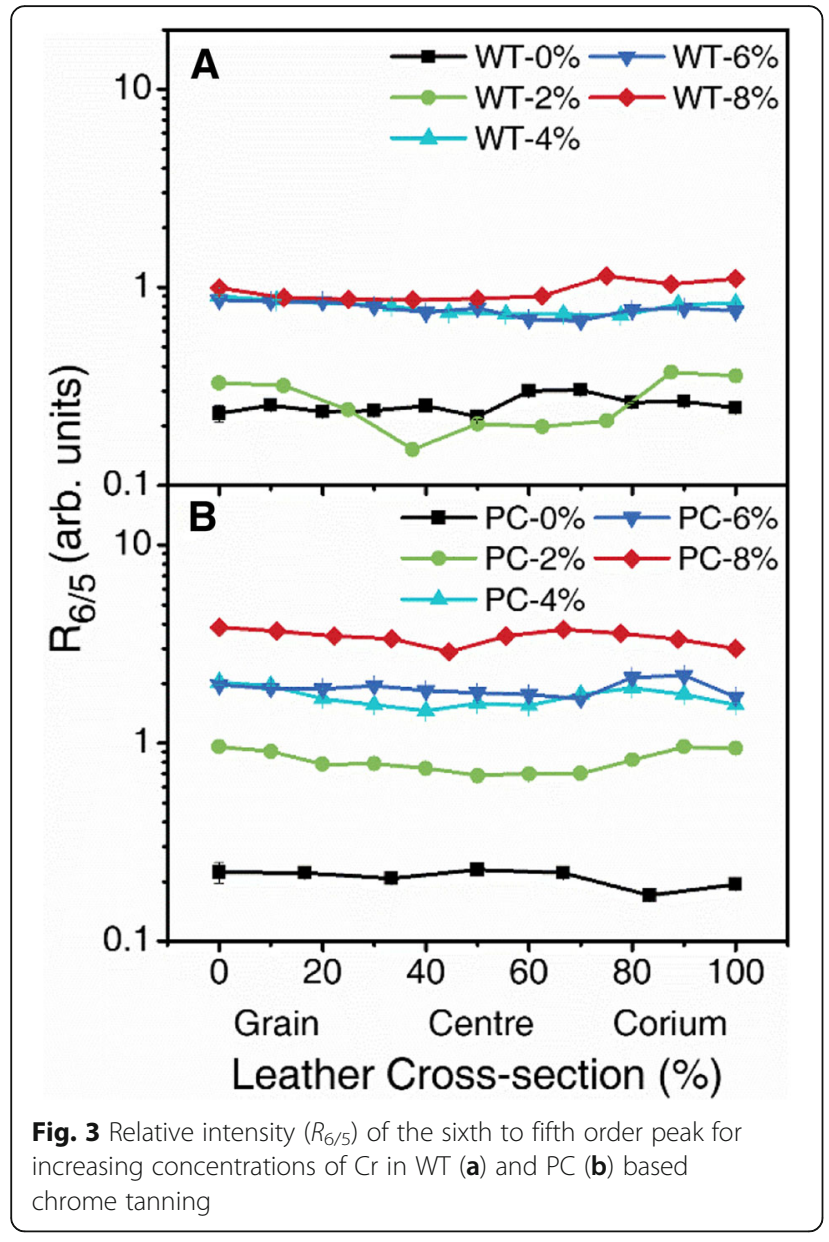

investigated. DSC experiments enabled us to study the denaturation temperatures of the leather samples. To highlight the changes in denaturation temperature through the cross-section, samples were basified to $\mathrm{pH}=$ 4 to facilitate the reaction of $\mathrm{Cr}$ with collagen. At low $\mathrm{pH}$ the samples were observed to have no increase in denaturation temperature compared to the pickled sample. For the WT samples, the onset temperature $\left(T_{d}\right)$ of collagen denaturation increased with increasing chrome concentration (Fig. 4a). Broad irregularly shaped endothermic peaks were observed for all WT samples, especially at lower concentrations of chrome. Broad peaks indicate a wide range of temperatures at which the endothermic event of collagen denaturation occur, highlighting the stabilisation effect by chrome tanning to various extents throughout the cross-section of leather. This can be attributed to the non-uniform penetration of $\mathrm{Cr}$ into the cross-section of the collagen matrix. The penetration of $\mathrm{Cr}$ through the leather cross-section is governed by the balance of diffusion and the binding of $\mathrm{Cr}$ to the collagen matrix, resulting in the centre of the hide having a lower $T_{d}$ compared to the surfaces. The peak width decreased as

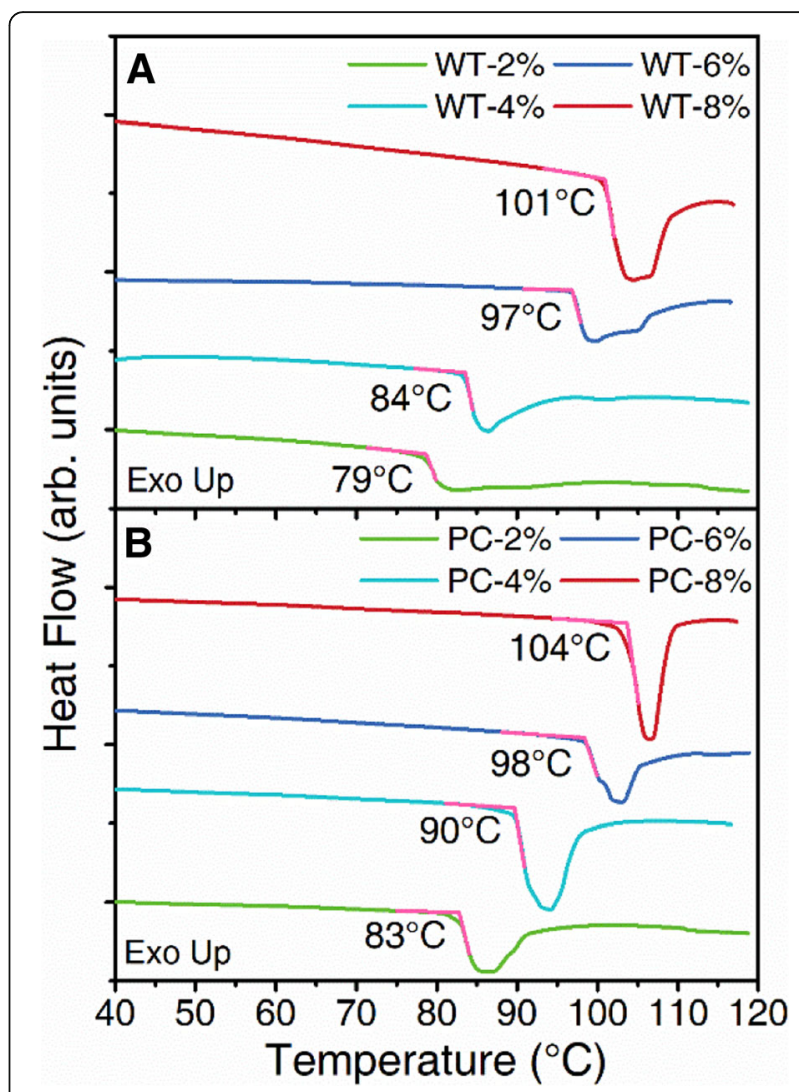

Fig. 4 DSC results for increasing concentrations of chrome in WT (a) and PC (b) based tanning. The denaturation temperatures of each sample are calculated and labelled alongside each curve

concentration increased, indicating more uniform penetration of $\mathrm{Cr}$ with increased chrome concentration. This was consistent with the $D$-period, which was found to be non-uniform across the leather cross-section for WT. The uniformity of both $T_{d}$ and $D$-period improved for WT samples as concentration of chromium sulphate increased.

For the PC samples, $T_{d}$ increased with chrome concentration (Fig. 4b). The PC samples showed slightly higher $T_{d}$ values than WT samples for the corresponding chrome concentrations. The endothermic peaks for the PC samples were also much sharper, indicating a more uniform penetration of $\mathrm{Cr}$ into the leather when PC was used. This was also consistent with the $D$-period which was relatively uniform across the leather crosssection.

\section{Conclusions}

Chrome tanned leather using PC had a significantly greater $\mathrm{Cr}$ uptake compared to standard leather and has the potential to replace water as the solvent of choice. SAXS analysis of the collagen structure showed an increase in $\mathrm{Cr}$ binding for PC samples, which was observed to drive the reduced hydration of the collagen 
triple helix. An even distribution of $\mathrm{Cr}$ through the collagen matrix was also observed in PC samples based on the D-period, supported by the uniform hydrothermal stability from DSC analysis. Fundamental understanding of the effects of recyclable solvents on the chrome tanning process could help reduce its environmental impact and lead to a more sustainable future for the leather industry.

\section{Additional file}

Additional file 1: Diffraction peak fitting method. (DOCX $22 \mathrm{~kb}$ )

\section{Abbreviations}

AAS: Atomic absorption spectrometry; DSC: Differential scanning calorimetry; PC: Propylene carbonate; PC-x\%: Hide tanned in PC using $x \%$ offer of basic chromium sulphate ( $\mathrm{x}=0$ is untanned); $R_{6 / 5}$ : The relative intensities of the 6th/5th order peaks; SAXS: Small-angle X-ray scattering; $T_{d}$ : Denaturation temperature; WT-x\%: Hide tanned in water using $\mathrm{x} \%$ offer of basic chromium sulphate $(x=0$ is untanned)

\section{Acknowledgements}

S.P., G.H., Y.Z., and J.B., thank the Ministry of Business, Innovation and Employment (MBIE) for providing funding through grant LSRX-1801. B.M., would like to acknowledge the Ministry of Science and Technology Taiwan (MOST) for postdoctoral research funding. Portions of this work were conducted on the SAXS beamline at NSRRC, Taiwan.

\section{Authors' contributions}

YZ, BM and SP contributed to the SAXS experiments and data analyses. JB and $Y Z$ conducted the DSC and AAS analyses. GH designed the tanning trials, and acquired data. All authors contributed equally to the writing of the manuscript. All authors read and approved the final manuscript.

\section{Funding}

The research is funded by the Ministry of Business, Innovation and Employment (MBIE) through grant LSRX-1801. The synchrotron SAXS beam time is funded by the Ministry of Science and Technology Taiwan (MOST).

\section{Availability of data and materials}

Detailed method for SAXS data analysis is included in the Additional file 1.

\section{Competing interests}

The authors declare that they have no competing interests.

\section{Author details}

'Leather and Shoe Research Association of New Zealand, P.O. Box 8094 Palmerston North 4472, New Zealand. ${ }^{2}$ Chemical Engineering Building, National Tsing Hua University, No. 101, Section 2, Guangfu Road, East District, Hsinchu City 300, Taiwan, Republic of China.

Received: 15 May 2019 Accepted: 13 August 2019

Published online: 21 August 2019

\section{References}

1. Bhargavi NR, Jayakumar GC, Sreeram KJ, Rao JR, Nair BU. Towards sustainable leather production: vegetable tanning in non-aqueous medium. J Am Leather Chem Assoc. 2015;110(4):97-102.

2. Sathish M, Madhan B, Sreeram KJ, Rao JR, Nair BU. Alternative carrier medium for sustainable leather manufacturing-a review and perspective. Clean Prod. 2016;112:49-58

3. Sathish M, Silambarasan S, Madhan B, Rao JR. Exploration of GSK'S solvent selection guide in leather industry: a CSIR-CLRI tool for sustainable leather manufacturing. Green Chem. 2016;18(21):5806-13.

4. Ding YQ, Chen $\mathrm{CL}, \mathrm{Gu}$ QR, Liao JM, Chuang PH. Application of molecular simulation to investigate chrome (III)-crosslinked collagen problems. Mode Simul Mater Sci Eng. 2014;22(3):035007.
5. Schroepfer M, Meyer M. DSC investigation of bovine hide collagen at varying degrees of crosslinking and humidities. Int J Biol Macromol. 2017; 103:120-8.

6. Kanagaraj J, Senthilvelan T, Panda RC, Kavitha S. Eco-friendly waste management strategies for greener environment towards sustainable development in leather industry: a comprehensive review. J Clean Prod. 2015;89:1-17.

7. Sathish M, Sreeram KJ, Raghava Rao J, Unni NB. Cyclic carbonate: a recyclable medium for zero discharge tanning. ACS Sustain Chem Eng. 2016;4(3):1032-40

8. Silvestre F, Rocrelle C, Rigal L, Gaset A. Optimum conditions for the reactivity of chromium (III) salts on collagen in the solvent tanning process. J Chem Technol Biotechnol. 1994;60(1):1-6.

9. Sathish M, Dhathathreyan A, Rao JR. Ultra-efficient tanning process: role of mass transfer efficiency and sorption kinetics of $\mathrm{Cr}$ (III) in processing of leather. ACS Sustain Chem Eng. 2019;7(4):3875-82.

10. Stone KR. Environmental profile for propylene carbonate. Washington DC: United States Environmental Protection Agency; 1998.

11. Covington AD. Tanning chemistry: the science of leather. Cambridge: Royal Society of Chemistry; 2009

12. Petruska JA, Hodge AJ. A subunit model for the tropocollagen macromolecule. New York: Proc Natl Acad Sci U S A. 1964;51(5):871-6.

13. Fratzl P. Collagen: structure and mechanics. New York: Springer; 2008.

14. Maxwell CA, Smiechowski K, Zarlok J, Sionkowska A, Wess TJ. X-ray studies of a collagen material for leather production treated with chromium salt. J Am Leather Chem Assoc. 2006;101(1):9-17.

15. Maxwell CA, Wess TJ, Kennedy CJ. X-ray diffraction study into the effects of liming on the structure of collagen. Biomacromolecules. 2006;7(8):2321-6.

16. Zhang Y, Ingham B, Cheong S, Ariotti N, Tilley RD, Naffa R, Holmes G, Clarke DJ, Prabakar S. Real-time synchrotron small-angle X-ray scattering studies of collagen structure during leather processing. Ind Eng Chem Res. 2017 . 57(1):63-9.

17. Zhang Y, Ingham B, Leveneur J, Cheong S, Yao Y, Clarke DJ, Holmes G, Kennedy J, Prabakar S. Can sodium silicates affect collagen structure during tanning? Insights from small angle X-ray scattering (SAXS) studies. RSC Adv. 2017:7(19):11665-71.

18. Zhang Y, Mansel BW, Naffa R, Cheong S, Yao Y, Holmes G, Chen HL, Prabakar S. Revealing molecular level indicators of collagen stability: minimizing chrome usage in leather processing. ACS Sustain Chem Eng. 2018:6(5):7096-104.

19. Zhang Y, Snow T, Smith AJ, Holmes G, Prabakar S. A guide to highefficiency chromium (III)-collagen cross-linking: synchrotron SAXS and DSC study. Int J Biol Macromol. 2019;126:123-9.

20. Bella J. Collagen structure: new tricks from a very old dog. Biochem J. 2016; 473(8):1001-25.

21. Bella J, Brodsky B, Berman HM. Hydration structure of a collagen peptide. Structure. 1995:3(9):893-906.

22. Fathima NN, Baias M, Blumich B, Ramasami T. Structure and dynamics of water in native and tanned collagen fibers: effect of crosslinking. Int J Biol Macromol. 2010;47(5):590-6.

23. Zhang C, Lin J, Jia X, Peng B. A salt-free and chromium discharge minimizing tanning technology: the novel cleaner integrated chrome tanning process. J Clean Prod. 2016;112:1055-63.

24. Yao Q, Chen H, Huang H, Liu B. Mechanism and effect of hydroxylterminated dendrimer as excellent chrome exhausted agent for tanning of pickled pelt. J Clean Prod. 2018;202:543-52.

25. Karthikeyan R, Balaji S, Chandrababu NK, Sehgal PK. Horn meal hydrolysatechromium complex as a high exhaust chrome tanning agent--pilot scale studies. Clean Techn Environ Policy. 2008;10(3):295-301.

26. Bünger MH, Foss M, Erlacher K, Hovgaard MB, Chevallier J, Langdahl B, Bünger C, Birkedal H, Besenbacher F, Pedersen JS. Nanostructure of the neurocentral growth plate: insight from scanning small angle $X$-ray scattering, atomic force microscopy and scanning electron microscopy. Bone. 2006:39(3):530-41.

27. Camacho NP, Rinnerthaler S, Paschalis EP, Mendelsohn R, Boskey AL, Fratzl P. Complementary information on bone ultrastructure from scanning small angle X-ray scattering and Fourier-transform infrared microspectroscopy. Bone. 1999:25(3):287-93.

28. Tomlin SG, Worthington CR. Low-angle X-ray diffraction patterns of collagen. Proceedings of the Royal Society of London. Series a. Math Phys Sci. 1956;235(1201):189-201. 
29. Fratzl P, Daxer A. Structural transformation of collagen fibrils in corneal stroma during drying. An x-ray scattering study. Biophys J. 1993;64(4):1210-4.

30. Stinson RH, Sweeny PR. Skin collagen has an unusual d-spacing. Biochimica et Biophysica Acta (BBA)-protein. Structure. 1980;621(1):158-61.

31. Wright BA. Low-angle X-ray diffraction pattern of collagen. Nature. 1948; 162(4105):23.

\section{Publisher's Note}

Springer Nature remains neutral with regard to jurisdictional claims in published maps and institutional affiliations.

Submit your manuscript to a SpringerOpen ${ }^{\circ}$ journal and benefit from:

- Convenient online submission

- Rigorous peer review

- Open access: articles freely available online

- High visibility within the field

- Retaining the copyright to your article

Submit your next manuscript at $\boldsymbol{\wedge}$ springeropen.com 